\title{
Combined posterior percutaneous pedicle screw fixation with delayed anterior monosegmental column reconstruction for unstable thoracolumbar burst fractures
}

\section{Chao Lou}

Lishui Hospital of Zhejiang University, the Fifth Affiliated Hospital of Wenzhou Medical University, Lishui Central Hospital

\section{Weiyang Yu}

Lishui Hospital of Zhejiang University, the Fifth Affiliated Hospital of Wenzhou Medical University, Lishui Central Hospital

\section{Zhenzhong Chen}

Lishui Hospital of Zhejiang University, the Fifth Affiliated Hospital of Wenzhou Medical University, Lishui Central Hospital

\section{Kangtao Jin}

Lishui Hospital of Zhejiang University, the Fifth Affiliated Hospital of Wenzhou Medical University, Lishui Central Hospital

\section{Jiawei Gao}

Lishui Hospital of Zhejiang University, the Fifth Affiliated Hospital of Wenzhou Medical University, Lishui Central Hospital

\section{Bin Pan}

Lishui Hospital of Zhejiang University, the Fifth Affiliated Hospital of Wenzhou Medical University, Lishui Central Hospital

\section{Feijun Liu}

Lishui Hospital of Zhejiang University, the Fifth Affiliated Hospital of Wenzhou Medical University, Lishui Central Hospital

\section{Dengwei He ( $\square$ hedw_spine@163.com )}

Affiliated Lishui Hospital of Zhejiang University, the Fifth Affiliated Hospital of Wenzhou Medical University, Lishui Central Hospital

\section{Research article}

Keywords: thoracolumbar burst fracture; combined approach; percutaneous; posterior pedicle screw fixation; anterior monosegmental reconstruction 
Posted Date: May 15th, 2020

DOI: https://doi.org/10.21203/rs.3.rs-27905/v1

License: (c) (i) This work is licensed under a Creative Commons Attribution 4.0 International License. Read Full License 


\section{Abstract}

Objective: This study aimed to assess the feasibility as well as the clinical and radiological outcomes of posterior percutaneous pedicle screw fixation (PPSF) combined with anterior monosegmental column reconstruction in unstable thoracolumbar burst fractures.

Methods: From January 2011 to August 2017, thirty-five patients with unstable thoracolumbar burst fractures were enrolled in this study. The patients underwent posterior PPSF combined surgery with delayed anterior monosegmental reconstruction utilizing titanium mesh cages. Clinical outcomes, radiological parameters, and treatment-related complications were assessed.

Results: The mean age of the patients was 44.8 years. The mean operative time and blood loss were 205 min and $560 \mathrm{ml}$, respectively. The mean follow-up period was 25.2 months. The Visual analog scale (VAS) pain score was significantly improved postoperatively, and the improvement was maintained until the final follow-up. The mean sagittal kyphosis was corrected from 16.3 preoperatively to 1.5 postoperatively, which increased slightly to 2.6 at the final follow-up. In 24 patients with neurologic dysfunction, 21 (87.5\%) patients had improvement after surgery. None obvious subsidence of the titanium mesh cage and none dislodgement, loosening or breakage of the instrumentation was observed in any patient during the follow-up period. Solid bony fusion was achieved in all patients.

Conclusions: Combined posterior PPSF with delayed anterior monosegmental column reconstruction for unstable thoracolumbar burst fractures can produce good clinical and radiological outcomes.

\section{Introduction}

Combined posterior-anterior stabilization for unstable thoracolumbar burst fractures has been advocated and successfully used [1-4]. The combined approach can provide the greatest stability, minimize the number of levels fused, and allow for a direct decompression of the spinal canal. There are numerous minimally invasive combined posterior-anterior techniques have attempted to decrease the associated morbidity. These have included combining vertebral augmentation with pedicle screw instrumentation [5, 6], transpedicular corpectomy with expandable cage combined with pedicle screw fixation [7], and minimally invasive corpectomy and posterior pedicle instrumentation [8, 9]. However, the optimal surgical treatment strategy for unstable thoracolumbar burst fractures remains controversial.

Unstable thoracolumbar burst fractures are characterized by loss of height of the anterior vertebral body, failure of the posterior tension band or retropulsion of fracture fragments into the spinal canal. However, vertebral body comminution often with the inferior endplate intact or shows a simple split fracture line only. Recently, some studies introduced the technique of anterior monosegmental reconstruction and reported good clinical and radiological outcomes for unstable thoracolumbar burst fractures [10, 11]. As opposed to bisegmental anterior column reconstruction, monosegmental reconstruction can spare one spinal motion segment and reduce the distance for osseous bridging to achieve bony fusion. Additionally, percutaneous pedicle screw fixation (PPSF) has rapidly gained acceptance with expanding indications [9, 
$12,13]$, and reported benefits include decreased muscle damage, less postoperative pain, and decreased blood loss [14]. However, there are no studies to date that have assessed the feasibility and outcomes of posterior PPSF combined with anterior monosegmental column reconstruction in unstable thoracolumbar burst fractures.

The aim of the present study, therefore, was to assess the feasibility as well as the clinical and radiological outcomes of posterior PPSF combined with anterior monosegmental column reconstruction in unstable thoracolumbar burst fractures.

\section{Materials And Methods Study Population}

We retrospectively reviewed all patients with traumatic unstable thoracolumbar burst fractures admitted to our institution from January 2011 to August 2017. The study was approved by the institutional review board and written informed consent was obtained from all individual participants included in the study. Inclusion criteria were defined as follows: (1) type B or type C according to the AOSpine Thoracolumbar Spine Injury Classification System [15]; (2) a score greater than 6 according to the load-sharing classification score [16]; (3) unnecessary for a patient to have a posterior spinal decompression, such as vertical lamina fracture; (4) the inferior endplate and the caudal intervertebral disc were confirmed to be intact or features only a single simple split fracture line by magnetic resonance imaging (MRI) and computed tomography (CT) scans. The exclusion criteria included two or more levels vertebral burst fractures, pathological or osteoporotic fracture, complete neurological injury, a history of previous spine surgery and severe multiple injuries.

\section{Surgical Technique}

All surgical procedures were performed by the same surgical group. The procedure of PPSF has been described in further detail in our previous study (Fig. 1) [17]. Using the percutaneous instruments, adequate distraction of the posterior column was performed and then instruments were locked. After that, we compressed the posterior column and distracted the anterior and central columns, and the screws were tightened. Posterior laminectomy and fusion were not performed in any patient. The anterior monosegmental column reconstruction was generally performed some time after the percutaneous posterior surgery. Details of the anterior monosegmental column reconstruction technique are well described in previous literature $[10,11]$. Briefly, the patient was placed in the right decubitus position and intubated with one-lung ventilation. In general, a left-sided approach performed through a retroperitoneal or extrapleural approach. After the burst vertebral body exposure, the cephalic disc was resected, and partial corpectomy of the vertebral body was performed to achieve complete canal decompression, while the lower portion of the vertebral body and the caudal disc was preserved. Then, the titanium mesh cage filled with cancellous bone from the partial corpectomy was monosegmentally implanted between the 
inferior endplate of the fractured vertebra and the inferior endplate of the cephalad intact vertebra. Anterior instrumentation fixation was not used in any patient. All the patients were mobilized at 3-5 days after the anterior surgery, and encouraged to walk or move with hyperextension braces, which was usually worn for about 2 months.

\section{Clinical And Radiographical Evaluations}

All patients were evaluated on admission, and at 1, 2, 3, 6, and 12 months postoperatively and then annually thereafter. Patient demographics, the mechanism and location of injury, duration from the initial injury to the posterior surgery, duration from the posterior surgery to the anterior surgery, time of followup, operative time, blood loss, and complications (related to either the posterior or the anterior approach) were investigated by reviewing the medical records. The level of pain was assessed using a Visual analog scale (VAS) of 0 to 10, with 10 indicating the most severe pain. Spinal cord injury was assessed using the American Spinal Injury Association (ASIA) impairment classification before surgery and at the final follow-up visit. The patients' work ability was determined at each time point.

All imaging measurements were obtained with a picture archiving and communication system (PACS) v3.0 (RADinfo, Hangzhou, China). Two independent experienced observers reviewed the imaging findings and consensus was reached when the initial reading of the two observers differed. The local kyphosis angle (LKA) was measured using the sagittal Cobb angle on the lateral radiograph, which was defined as the angle between the cranial endplate of the superior vertebra and the caudal endplate of the inferior vertebra to the fractured vertebra. Subsidence of the titanium mesh cage was observed during the followup period. Bony fusion was evaluated using reconstructed CT scans and flexion-extension radiographs. A fusion was confirmed by a progressive increase in interspace bone density and blurring of the adjacent endplate, as well as by the presence of continuous bony bridge bone on flexion-extension radiographs and CT scans.

\section{Statistical analysis}

The statistical analysis was performed with the SPSS software 18.0 (SPSS Inc, Chicago, IL, USA). Metric scaled data are reported as arithmetic mean \pm standard deviation and categorical data as absolute frequency and percentage distribution. Paired student's t test was used to compare the difference between preoperative and postoperative results. Statistical significance was set at $P<0.05$.

\section{Results}

\section{Patient characteristics}

Thirty-five patients with unstable thoracolumbar burst fractures underwent posterior PPSF with delayed anterior monosegmental column reconstruction surgery at the authors' institution. Typical patient images were displayed in Figs. 2. As demonstrated in Table 1, the mean age of the patients was $44.8 \pm 12.3$ 
(range 31-58) years; the sample comprised 26 men and 9 women. The causes of injury were falling from a height in 22 patients, motor vehicle accident in 9 patients and a blunt contusion from a heavy falling object in 4 patients. Eight patients presented with other traumatic injuries. The burst fracture was at L2 in 16 patients, L1 in 12, T12 in 4, T11 in 1, and L3 in 2. According to the AOSpine Thoracolumbar Spine Injury Classification System, type B in 29 patients, and type $C$ in 6 patients. Vertebral body fracture classified according to the AOSpine Thoracolumbar Spine Injury Classification System was subtype A3 in 28 patients, and subtype A4 in 7 patients. The mean load sharing score was $8.1 \pm 0.9$ (range 7-9). The mean follow-up was $25.2 \pm 14.1$ (range 18-68) months. 
Table 1

Baseline characteristics of 35 patients

\section{Characteristics}

Age (years)

Sex (male)

Mechanism of injury

Fall from height

Motor vehicle accident

Other

Level of fracture

$\mathrm{T} 11$

$\mathrm{T} 12$

L1

L2

L3

AOSpine type

Type B

Type C

AOSpine VB

A3

A4

Load sharing score

Follow-up time (months)
$44.8 \pm 12.3 \quad 31-58$

26

22

9

4

1

4

12

16

2

29

7-9

6

28

7

$8.1 \pm 0.9$

$-$

-

-

-

- -

16 -

-

(n)

8




\section{Characteristics}

Time from the initial injury to the first surgery(days)

Time from the first surgery to the second surgery(days)

Length of hospitalization(days)

Posterior operative time(min)

Posterior blood loss(ml)

Anterior operative time(min)

Anterior blood loss $(\mathrm{ml})$

Complications

Transient pulmonary complications

Transient paralytic ileus

Dysesthesia
$3.8 \pm 2.4$

$6.3 \pm 2.9$

$20.5 \pm 11.7$

$85 \pm 15$

$50 \pm 25$

$124 \pm 28$

$510 \pm 185$

3

2

2
2-8

$4-10$

$14-32$

$60-120$

$30-80$

$90-160$

$300-800$

AOSpine type, injury type according to the AOSpine Thoracolumbar

Spine Injury Classification System; $A O S p i n e$ VB vertebral body (VB) fracture classified according to the AOSpine Thoracolumbar Spine Injury Classification System

Table 2

Clinical and radiological results

\begin{tabular}{|lll|}
\hline Parameter & Preoperation & Postoperation Final follow-up \\
\hline VAS pain score & $8.9 \pm 1.2$ & $3.5 \pm 0.42 .6 \pm 0.5$ \\
\hline ODI score & $91.6 \pm 8.5$ & $28.5 \pm 9.322 .7 \pm 7.8$ \\
\hline LKA & $17.6 \pm 12.3$ & $3.2 \pm 1.84 .7 \pm 2.1$ \\
\hline $\begin{array}{l}\text { Values are presented as the mean } \pm \text { SD; VAS, Visual analog scale; ODI, Oswestry disability index; LKA, } \\
\text { Local kyphosis angle. }\end{array}$ & \\
\hline
\end{tabular}

\section{Clinical Results}

The mean posterior operative time and blood loss were $85 \pm 15$ (range 60-120) min and $50 \pm 25$ (range 30-80) $\mathrm{ml}$, respectively. The mean anterior operative time and blood loss were $124 \pm 28$ (range 90-160) min and $510 \pm 185$ (range 300-800) $\mathrm{ml}$, respectively. The mean duration from the initial injury to the first surgery was $3.8 \pm 2.4$ (range 2-8) days; the mean duration from the first surgery to the second surgery was $6.3 \pm 2.9$ (range $4-10$ ) days. The mean length of hospitalization was $20.5 \pm 11.7$ (range, 14-32) days. Complications occurred in 7 patients. There were three cases of transient pulmonary complications, two cases of transient paralytic ileus, and two of dysesthesia that all cases could be treated 
conservatively. No patients experienced wound infection, intraoperative neurovascular injury, or cagerelated complications requiring revision surgery.

The mean VAS pain score was improved from $8.6 \pm 1.2$ points preoperatively to $3.1 \pm 0.4$ points postoperatively $(P=0.028)$. At the final follow-up visit, the mean VAS pain score was $2.5 \pm 0.4$ points, which demonstrated no significant variation compared with the postoperative VAS pain score $(P=0.093)$.

According to the ASIA impairment classification, the preoperative neurological function was graded as B in 2 patients, $C$ in 6 patients, $D$ in 16 patients, and $E$ in 11 patients. At the final follow-up visit, the neurological function was recorded as follows: $B$ in 1 patient, $C$ in 2 patients, $D$ in 6 patients, and $E$ in 26 patients. In 24 patients with preoperative neurologic dysfunction, 21 (87.5\%) patients showed improvement after surgery.

Eighteen patients returned to work within 6 months and a further 12 returned to work subsequently. At the time of the final follow-up, 20 of 30 patients had returned to a similar job, and 10 patients changed to a less physically demanding job.

\section{Radiological Outcome}

Preoperatively, the mean LKA was $16.3 \pm 12.7$ (range, -3.4 to 28.2) degrees. Through surgical treatment, the mean LKA was reached to $1.5 \pm 7.3$ (range, -10.5 to 10.3) degrees, which has a significant correction $(P<0.001)$. At the final follow-up, the mean LKA was $2.6 \pm 5.2$ (range, -5.8 to 13.8$)$ degrees. No significant differences were found between postoperatively and at the final follow-up visit $(P=0.082)$. Bony fusion of the titanium mesh cage was eventually achieved in all patients at mean $10.5 \pm 2.7$ months postoperatively. None obvious subsidence of the titanium mesh cage and none dislodgement, loosening or breakage of the instrumentation requiring revision surgery was observed in any patient during the follow-up period.

\section{Discussion}

Unstable thoracolumbar burst fractures are common spinal injury frequent results severe spinal canal compromise, collapse of anterior and middle spinal columns, a significant posterior element lesion, severe neurological injury and local kyphosis deformity. Regardless of the management strategy, the conventional major goals of treatment are typically early fracture stabilization, reduction of neurologic deficit and correction of kyphotic deformity. However, recently, some update goals of treatment have been recognized including minimally invasive surgery and limiting the motion segments fused. Following this principle, several studies have reported favorable outcomes with PPSF or monosegmental anterior reconstruction for selected thoracolumbar fractures [10-12, 27, 28]. However, there are no studies on combined posterior PPSF with anterior monosegmental column reconstruction in unstable thoracolumbar burst fractures. This study is the first report systematically evaluating the feasibility and outcome of posterior PPSF with delayed anterior monosegmental column reconstruction for unstable thoracolumbar 
burst fractures. Our study found that the clinical and radiographical outcomes were significantly improved postoperatively and maintained well at the final follow-up. Especially no major complications requiring revision surgery during follow-up, indicating that this technique is a valuable surgical option for unstable thoracolumbar burst fractures.

Currently, most authors prefer an anterior-only or posterior-only approach surgical technique to treat unstable thoracolumbar burst fractures and good results had been reported [17-20]. Hitchon et al. [21] reported that a $5 \%(2 / 38)$ failure rate treated by anterior instrumentation alone and a repeated surgery that involved posterior instrumentation-augmented with fusion was required, and a rate of $8 \%(2 / 25)$ with posterior instrumentation complications necessitating repeated operation in unstable thoracolumbar burst fractures. In the study about anterior decompression and stabilization reported by Kaneda et al. [22], the failure rate was $7 \%(10 / 150)$ with pseudarthrosis necessitating posterior instrumentation and fusion. McAfee et al. [23] reported the failure rate was $6 \%(2 / 35)$ when the Kaneda dual rod-screw construct was used. Sasso et al. [24] reported a statistical significance loss (averaged 8.1 degrees) of the sagittal LKA in posterior-only short-segment group at follow-up. Though a posterior long segmental fixation and fusion can provide more rigid support, it reduces the range of motion and leads to troubles in the activities of daily living $[25,26]$.

To overcome the disadvantages of anterior-only and posterior-only approach, many authors have reported satisfactory results with a combined anterior-posterior approach [8-11]. However, it was generally believed that the conventional combined anterior-posterior approach has more surgical invasiveness and morbidity than those of an anterior-only or posterior-only approach. Recently, a few studies $[27,28]$ have combined posterior pedicle instrumentation with percutaneous augmented stabilization of the anterior column, yielding encouraging preliminary results in osteoporotic and younger patients with unstable thoracolumbar burst fractures, however, some certain concerns arise from this technique include the long-term outcomes was unclear, the cement-related complications, the concern pertains to the use of cement in younger patients. This study presents another potential surgical technique with less morbidity, blood loss, paraspinal muscle damage and pain. PPSF is increasingly widespread in the spine surgery and gains rapid acceptance with expanding indications $[12,29]$. PPSF was performed by sparing the paravertebral musculature and avoiding damage to the zygapophysial joint. Some studies have demonstrated that percutaneous procedures were superior in terms of iatrogenic soft-tissue injury, incision size, blood loss, postoperative pain, and the length of hospitalization compared open procedures, which make rehabilitation easier and faster [30-32]. Previous studies have quantified the amount of muscle injury during surgical exposure of the spine with measurements of serum creatine kinase $[33,34]$. Percutaneous procedures were found to result in significantly lower levels of serum creatine kinase than open lumbar fusions. Disadvantages of this technique include exposed the spine surgeon, staff, and patient to significantly greater radiation levels, as well as an initially longer operative time during the learning curve compared open procedures [35]. In addition, because of corpectomy, an anterior column reconstruction performed within the first few days after the traumatic event, generally results in significant blood loss [18, 34]. In study by McDonough et al. [18], an average blood loss of $1750 \mathrm{ml}$ was reported for patients who underwent surgery within 24 hours after admission. In study by 
Carl et al. [36], an average blood loss of $2300 \mathrm{ml}$ and an average of a 4-day delay until surgery after the accident were reported. However, Tofuku et al. [37] reported an average blood loss of $545 \mathrm{ml}$ in combined delayed staged anterior surgery. In the present study, the average blood loss was $516 \mathrm{ml}$ in combined delayed staged anterior surgery, which was more favorable and less invasive than the conventional onestage combined procedure, and also comparable with that of Tofuku et al. [37].

In contrast to the conventional anterior reconstruction, the lower portion of the injured vertebral body and the caudal disc was preserved during anterior monosegmental reconstruction in our patients. Monosegmental reconstruction can spare one spinal motion segment and reduce the distance of osseous bridging to achieve bony fusion. Lindtner et al. [10] reported satisfactory clinical and radiological outcomes of monosegmental reconstruction with a mean correction of $-15.6 \pm 7.7^{\circ}$ and postoperative loss of correction averaged $2.7 \pm 2.7^{\circ}$. Lai et al. [11] reported the mean sagittal LKA was corrected from 21.2 preoperatively to 2.5 postoperatively, and increased slightly to 4.3 at the final follow-up. In our case series, LKA correction was also effectively improved postoperatively and maintained during 25.2 months of follow-up. Lai et al. [11] reported that the mean operative time and blood loss were $230 \mathrm{~min}$ and $645 \mathrm{ml}$, respectively, with the monosegmental anterior reconstruction combined with conventional open posterior surgery, whereas the mean operative time and blood loss were $205 \mathrm{~min}$ and $560 \mathrm{ml}$, respectively, in our series. Posterior instrumentation removal after monosegmental anterior reconstruction, however, is essential to restore mobility in the nonfused segment and to provide the patients with the potentially beneficial effects of sparing one motion segment, although this was not evaluated in this study. We routinely recommended implant removal to our patients after monosegmental anterior reconstruction, however, half of the patients refused removal. Another some patients have not come back for posterior implant removal during our follow-up. Further studies needed to be carried out to investigate the value of monosegmental anterior reconstruction and the potential additional risks of posterior implant removal.

One of the concerns regarding the anterior monosegmental reconstruction technique was titanium mesh cage subsidence since the loading surface of the residual vertebral body was cancellous bone. Lindtner et al. [10] analyzed the incidence of subsidence treated by combined posterior-anterior stabilization and either anterior monosegmental (18 patients) or bisegmental (19 patients) reconstruction using the expandable vertebral body replacement device. Subsidence were observed in 5 patients after anterior monosegmental but none after bisegmental reconstruction and two risk factors for subsidence with anterior monosegmental reconstruction were proposed: positioning the titanium mesh cage onto the weak cancellous bone as not close enough to the inferior endplate and onto a sort of "free floating" inferior endplate fragment created by the presence of multiple fracture lines. We have initially realized these pitfalls, thus the titanium mesh cage was monosegmentally implanted close enough to the inferior endplate of the cephalad intact vertebra and one of the inclusion criteria was the inferior endplate and the caudal intervertebral disc were confirmed to be intact or features only a single simple split fracture line by MRI and CT scans. In present study, however, bony fusion was achieved in all patients, and none obvious subsidence of the titanium mesh cage. In addition, because of the anterior monosegmental reconstruction, lumbar motion would be theoretically improved after posterior instrumentation removal, although this was not evaluated in this study.

Page 11/18 
This study had several potential limitations. The study was retrospective by design. In addition, the study group was relatively small, limiting its statistical power. Furthermore, these findings are based on a retrospective chart review, which lacks a comparison group. Further randomized studies with a larger group and a comparison group would be helpful in terms of evaluating this issue.

\section{Conclusions}

Combined posterior PPSF with delayed anterior monosegmental column reconstruction for unstable thoracolumbar burst fractures can produce good clinical and radiological outcomes. As long as the indications were correctly chosen, this minimally invasive procedure could be a valuable surgical option for the surgical treatment of unstable thoracolumbar burst fractures.

\section{Abbreviations}

ASIA- American Spinal Injury Association

CT - Computed tomography

LKA-Local kyphosis angle

MRI - Magnetic resonance imaging

PPSF- Percutaneous pedicle screw fixation

VAS - Visual analog scale

\section{Declarations}

\section{Acknowledgements}

No

\section{Ethical review committee statement}

Ethical approval was obtained from the Medical Ethics Committee of our hospital. Additionally, all patients gave written informed consent for their information to be stored in the hospital's database and used for research.

\section{Authors' contributions}

All authors were involved in conception and design. CL, WYY, FJL, and DWH contributed to study design. ZZC, KTJ, and JWG contributed to study conduct. WYY, FJL, and BP contributed to data collection and analysis. CL, FJL, and DWH contributed to data interpretation. CL, WYY, and DWH contributed to drafting 
of the manuscript. All authors take responsibility for the integrity of the data analysis. All authors read and approved the final manuscript.

\section{Funding}

This study was partially supported by the Zhejiang Province Natural Science Foundation of China (LY17C100002), the Zhejiang Experimental Animal and Technology Program Foundation of China (2018C37099), the Zhejiang Province Scientifc Project of Health and Medicine of China (2018KY936).

\section{Availability of data and materials}

The datasets used and analyzed during the current study are available from the corresponding author on reasonable request.

\section{Ethics approval and consent to participate}

The study was reviewed and approved by the institutional review board and the ethics committee of our institution. Patients or their family members agreed to our study, and signed the informed consents.

\section{Consent for publication}

Not applicable

\section{Competing interests}

The authors declare that they have no competing interests

\section{References}

1. Schnake KJ, Stavridis SI, Kandziora F. Five-year clinical and radiological results of combined anteroposterior stabilization of thoracolumbar fractures. J Neurosurg Spine. 2014;20(5):497-504.

2. Knop C, Kranabetter T, Reinhold M, Blauth M. Combined posterior-anterior stabilisation of thoracolumbar injuries utilising a vertebral body replacing implant. Eur Spine J. 2009;18(7):949-963.

3. Lange U, Edeling S, Knop C, Bastian L, Oeser M, Krettek C, et al. Anterior vertebral body replacement with a titanium implant of adjustable height: a prospective clinical study. Eur Spine J. 2007;16(2):161-172.

4. Ray WZ, Krisht KM, Dailey AT, Schmidt MH. Clinical outcomes of unstable thoracolumbar junction burst fractures: combined posterior short-segment correction followed by thoracoscopic corpectomy and fusion. Acta Neurochir (Wien). 2013;155(7):1179-1186.

5. Korovessis P, Hadjipavlou A, Repantis T. Minimally invasive short posterior instrumentation plus balloon kyphoplasty with calcium phosphate for burst and severe compression lumbar fractures. Spine (Phila Pa 1976). 2008;33(6):658-667. 
6. Pflugmacher R, Agarwal A, Kandziora F, Klostermann C. Balloon kyphoplasty combined with posterior instrumentation for the treatment of burst fractures of the spine: 1-year results. J Orthop Trauma. 2009;23(2):126-131.

7. Hu W, Wang B, Run H, Zhang X, Wang Y. Pedicle subtraction osteotomy and disc resection with cage placement in post-traumatic thoracolumbar kyphosis, a retrospective study. J Orthop Surg Res. 2016;11(1):112.

8. Theologis AA, Tabaraee E, Toogood P, Kennedy A, Birk H, McClellan RT, et al. Anterior corpectomy via the mini-open, extreme lateral, transpsoas approach combined with short-segment posterior fixation for single-level traumatic lumbar burst fractures: analysis of health-related quality of life outcomes and patient satisfaction. J Neurosurg Spine.2016; 24(1):60-68.

9. Eck JC. Minimally invasive corpectomy and posterior stabilization for lumbar burst fracture. Spine J.2011; 11(19):904-908.

10. Lindtner RA, Mueller M, Schmid R, Spicher A, Zegg M, Kammerlander C, et al. Monosegmental anterior column reconstruction using an expandable vertebral body replacement device in combined posterior-anterior stabilization of thoracolumbar burst fractures. Arch Orthop Trauma Surg.2018;138(7): 939-951.

11. Lai O, Hu Y, Yuan Z, Sun X, Dong W, Zhang J, Zhu B. Modified one-stage posterior/anterior combined surgery with posterior pedicle instrumentation and anterior monosegmental reconstruction for unstable Denis type B thoracolumbar burst fracture. Eur Spine J.2017;26(5):1499-1505.

12. Fuentes S, Blondel B, Metellus P, Gaudart J, Adetchessi T, Dufour H. Percutaneous kyphoplasty and pedicle screw fixation for the management of thoraco-lumbar burst fractures. Eur Spine $J$. 2010;19(8):1281-1287.

13. Harris EB, Massey P, LawrenceJ, Rihn J, Vaccaro A, Anderson DG. Percutaneous techniques for minimally invasive posterior lumbar fusion. Neurosurg Focus. 2008;25(2):E12.

14. Dickerman RD, East JW, Winters K, Tackett J, Hajovsky-Pietla A. Anterior and posterior lumbar interbody fusion with percutaneous pedicle screws: comparison to muscle damage and minimally invasive techniques. Spine (Phila Pa 1976). 2009;34(25):E923-E925.

15. Vaccaro AR, Oner C, Kepler CK, Dvorak M, Schnake K, Bellabarba C, et al. AOSpine thoracolumbar spine injury classification system: fracture description, neurological status, and key modifiers. Spine (Phila Pa 1976). 2013;38(23):2028-2037.

16. McCormack T, Karaikovic E, Gaines RW. The load sharing classification of spine fractures. Spine (Phila Pa 1976). 1994;19(15):1741-1744.

17. He D, Wu L, Sheng X, Xiao Q, Zhu Y, Yu W, et al. Internal fixation with percutaneous kyphoplasty compared with simple percutaneous kyphoplasty for thoracolumbar bürst fractures in elderly patients: a prospective randomized controlled trial. Eur Spine J. 2013;22(10):2256-2263.

18. McDonough PW, Davis R, Tribus C, Zdeblick TA. The management of acute thoracolumbar burst fractures with anterior corpectomy and Z-plate fixation. Spine (Phila Pa 1976). 2004;29(17):19011908. 
19. Xu JG, Zeng BF, Zhou W, Kong WQ, Fu YS, Zhao BZ, et al. Anterior Z-plate and titanic mesh fixation for acute burst thoracolumbar fracture. Spine (Phila Pa 1976). 2011;36(7):E498-E504.

20. Zahra B, Jodoin A, Maurais G, Parent S, Mac-Thiong JM. Treatment of thoracolumbar burst fractures by means of anterior fusion and cage. J Spinal Disord Tech. 2012;25(1):30-37.

21. Hitchon PW, Torner J, Eichholz KM, Beeler SN. Comparison of anterolateral and posterior approaches in the management of thoracolumbar burst fractures. J Neurosurg Spine. 2006;5(2):117-125.

22. Kaneda K, Taneichi H, Abumi K, Hashimoto T, Satoh S, Fujiya M. Anterior decompression and stabilization with the Kaneda device for thoracolumbar burst fractures associated with neurological deficits. J Bone Joint Surg Am. 1997;79(1):69-83.

23. McAfee PC. Complications of anterior approaches to the thoracolumbar spine: Emphasis on Kaneda instrumentation. Clin Orthop Relat Res. 1994;306:110-119,

24. Sasso RC, Renkens K, Hanson D, Reilly T, McGuire RA Jr, et al. Unstable thoracolumbar burst fractures: anterior-only versus short-segment posterior fixation. J Spinal Disord Tech.2006;19(4):242248.

25. McLain RF. The biomechanics of long versus short fixation for thoracolumbar spine fractures. Spine (Phila Pa 1976). 2006;31 (11 Suppl):S70- S79.

26. Tezeren G, Kuru I. Posterior fixation of thoracolumbar burst fracture: short-segment pedicle fixation versus long-segment instrumentation. J Spinal Disord Tech. 2005;18(6): 485-488.

27. Marco RA, Kushwaha VP. Thoracolumbar burst fractures treated with posterior decompression and pedicle screw instrumentation supplemented with balloon-assisted vertebroplasty and calcium phosphate reconstruction. J Bone Joint Surg Am. 2009; 91(1): 20-28.

28. Rahamimov N, Mulla H, Shani A, Freiman S. Percutaneous augmented instrumentation of unstable thoracolumbar burst fractures. Eur Spine J. 2012;21(5): 850-854.

29. Ni WF, Huang $Y X, C h i$ YL, Xu HZ, Lin $Y$, Wang $X Y$, et al. Percutaneous pedicle screw fixation for neurologic intact thoracolumbar burst fractures. J Spinal Disord Tech. 2010;23(8):530-537.

30. Rampersaud YR, Annand N, Dekutoski MB. Use of minimally invasive surgical techniques in the management of thoracolumbar trauma: current concepts. Spine (Phila Pa 1976). 2006;31 (11 Suppl):S96-S102.

31. Smith JS, Ogden AT, Fessler RG. Minimally invasive posterior thoracic fusion. Neurosurg Focus. 2008;25(2):E9.

32. Dickerman RD, East JW, Winters K, Tackett J, Hajovsky-Pietla A. Anterior and posterior lumbar interbody fusion with percutaneous pedicle screws: comparison to muscle damage and minimally invasive techniques. Spine (Phila Pa 1976). 2009;34(25):E923-E925.

33. Arts MP, Nieborg A, Brand R, Peul WC. Serum creatine phosphokinase as an indicator of muscle injury after various spinal and nonspinal surgical procedures. J Neurosurg Spine. 2007;7(3): 282-286.

34. Kumbhare D, Parkinson W, Dunlop B. Validity of serum creatine kinase as a measure of muscle injury produced by lumbar surgery. J Spinal Disord Tech. 2008;21(1):49-54. 
35. Bindal RK, Glaze S, Ognoskie M, Tunner V, Malone R, Ghosh S. Surgeon and patient radiation exposure in minimally invasive transforaminal lumbar interbody fusion. J Neurosurg Spine. 2008;9(6):570-573.

36. Carl AL, Tranmer BI, Sachs BL. Anterolateral dynamized instrumentation and fusion for unstable thoracolumbar and lumbar burst fractures. Spine (Phila Pa 1976). 1997;22(6):686-690.

37. Tofuku K, Koga H, ljiri K, Ishidou Y, Yamamoto T, Zenmyo M, et al. Combined posterior and delayed staged mini-open anterior short-segment fusion for thoracolumbar burst fractures. J Spinal Disord Tech.2012;25(1): 38-46.

\section{Figures}



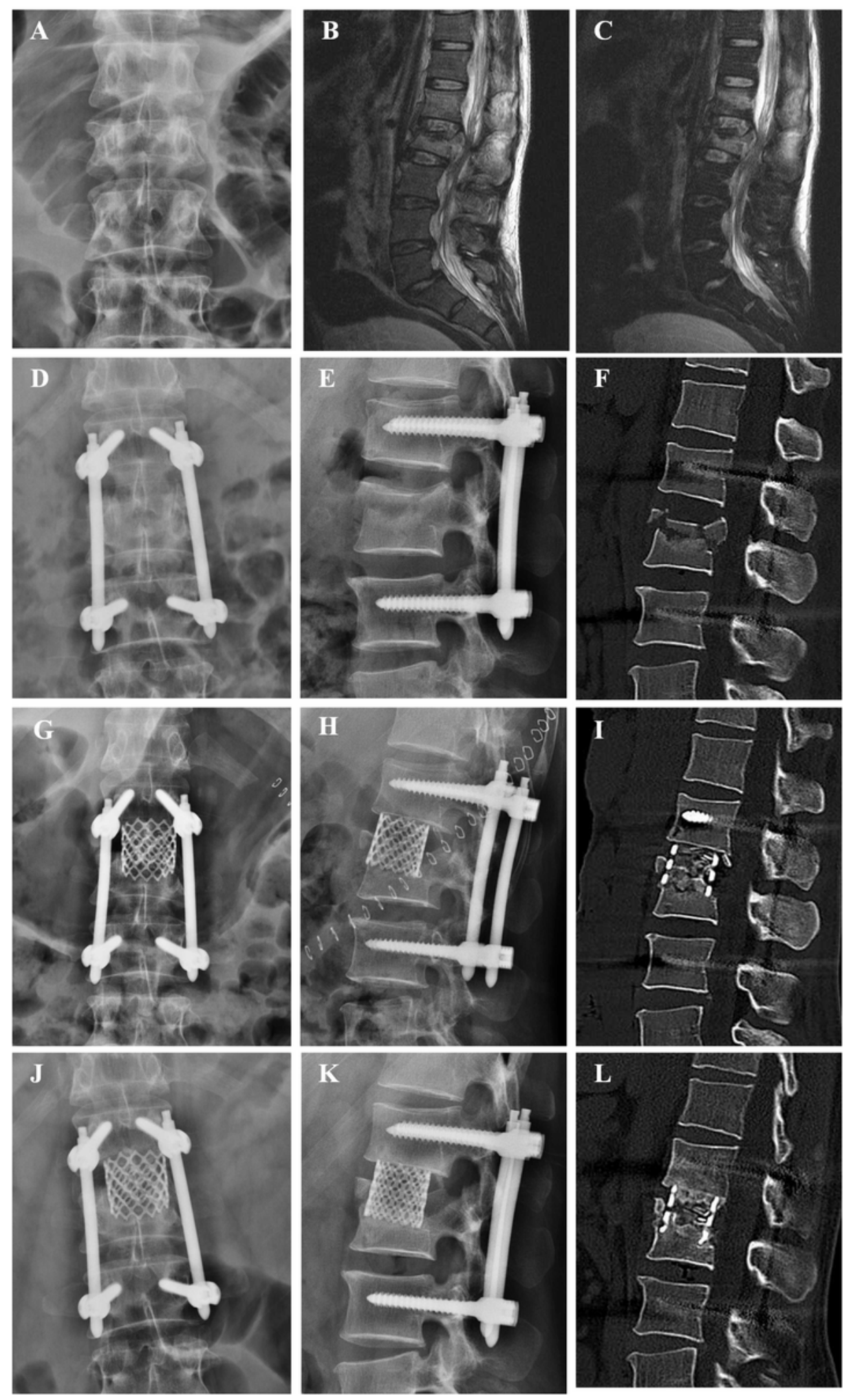

Figure 1

Diagram showing skin incision design for posterior percutaneous pedicle screw fixation 


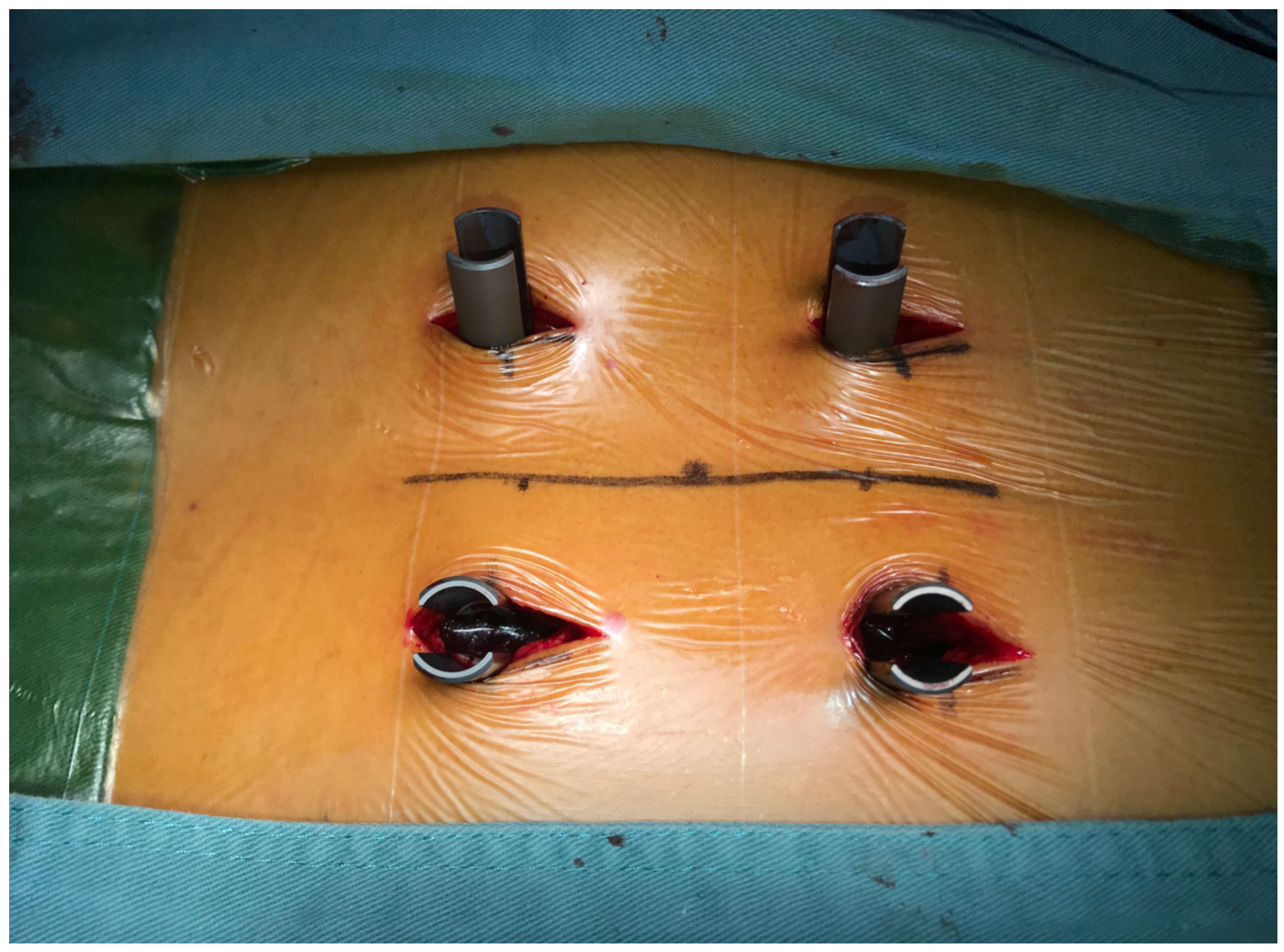

Figure 2

A-C Preoperative plain radiograph and sagittal MRI images showing L2 AOSpine subtype B2 fracture. D-I Immediate postoperative plain radiographs and sagittal CT images of the same patient showing the characteristics of this modified combined surgery. J-L Plain radiographs and sagittal CT images of the same patient at the final follow-up showing that the position of the titanium mesh cage was well maintained and a continuous bony bridge was observed. 